\title{
A Neural Network Based Adaptive Sliding Mode Controller: Application to a Power System Stabilizer
}

\author{
Hussain N. Al-Duwaish ${ }^{a^{*}}$ and Zakariya M. Al-Hamouz, ${ }^{a, 1}$ \\ ${ }^{a}$ Department of Electrical Engineering, King Fahd University of Petroleum \& Minerals, \\ Dhahran 31261, Saudi Arabia \\ *Corresponding Author- e-mail: hduwaish@kfupm.edu.sa, Tel: +966 3860 2747, Fax: +966 38603535 \\ 1e-mail: zhamouzh@kfupm.edu.sa, Tel: +9663860 2782, Fax: +966 38603535
}

Abstract - In this paper, a neural networks (NN) based adaptive sliding mode controller (SMC) is introduced. The selection of SMC feedback gains is normally based on one operating point and thus the performance of the controller away from the design operating point is, of necessity, a compromise. The Adaptive SMC is proposed to overcome the limitations imposed on the effectiveness of the SMC under different operating conditions. Neural networks are used for on-line prediction of the optimal SMC gains when the operating point changes. The proposed method has been applied to a power system stabilizer (PSS) of a single machine power system. Simulation results are included to demonstrate the performance of the proposed control scheme.

Key Words: Neural networks, sliding mode control, power system stabilizers, Genetic algorithms.

\section{INTRODUCTION}

The SMC is essentially a switching feedback control where the gains in each feedback path switch between two values according to some rule. The switching feedback law drives the controlled system's state trajectory onto specified surface called the sliding surface which represents the desired dynamic behavior of the controlled system. The advantage of switching between different feedback structures is to combine the useful properties of each 
structure and to introduce new properties that are not present in any of the structures used [1-3]. SMC has been reported as one of the most effective control methodologies for nonlinear power system applications due to its disturbance rejection, strong robustness subject to system parameter variations, uncertainties and external disturbances [1-3]. These applications include: rectifier/inverter motor drive [4], synchronous motor [5], wind energy systems [6], load frequency control [7], power system stabilizers [8], and static VAR compensators [9] . Conventionally, the design procedure of SMC involves the design of a suitable switching surface that will guarantee a stable behavior of the system and the design of a suitable feedback gains. However, one of the main problems of SMC design is the selection of the feedback gains which are generally selected by trial and error such that they will satisfy certain system performance requirements [1-3]. In all of the above applications [4-9], the feedback gains are fixed and selected by trial and error.

To solve the problem of feedback gains selection, a diagonalization method was proposed in [10]. The diagonalization method involves conversion of multi-input design problem into single input design problems and constructing new control vector via a nonsingular transformation of the original control vector. This will add to the complexity of controller design as it requires detailed analytical derivation to satisfy the conditions for the existence of a sliding mode. This problem has also been considered in [11] where all allowable values of the feedback gains are tried and a performance index for each possible set of feedback gains is evaluated. The optimal feedback gains selected are those which minimize the performance index. This approach is numerically intensive especially for large numbers of feedback gains. Moreover, the methods proposed in $[10,11]$ employed fixed feedback gains for all operating points.

Recently, the design of SMC feedback gains has been formulated an optimization problem where search optimization algorithms are used [12-14]. In this way, the optimum settings of the SMC applied to power system control problems can be found even with the presence of nonlinearities in the model. This method provides a systematic way of arriving at the optimal values for feedback gains of the SMC. In [12], genetic algorithms have 
been used to select the optimal feedback gain for load frequency control problem. Tabu search has been used in [13] for the selection of SMC feedback gains for multi-area nonlinear load frequency control. For a nonlinear single machine infinite system, particle swarm optimization has been used to select the feedback gains of the SMC controller [14]. The feedback gains selection of the SMC proposed in [12-14] is based on one operating point which results in fixed SMC gains for the entire operating points. Therefore, the performance of the controller away from the design operating point is, of necessity, a compromise.

The limitations imposed on the effectiveness of the SMC by different operating conditions can be overcome by using adaptive control techniques. In this paper, an adaptive sliding mode controller (SMC) using neural networks is proposed to enhance the performance under different operating points. The relationship between the system operating point and the SMC controller gains is highly nonlinear. Neural networks are known for their universal nonlinear approximation capabilities [15]. Therefore, the authors propose the use of NN to perform an on line nonlinear mapping between the operating points as input and SMC gain as output. The adaptive SMC will include a neural network which is trained off-line to update the feedback gains when the operating point changes. The training data for the neural networks (NN) is generated off-line using genetic algorithms following the procedure described in author's previous work [12]. To demonstrate the applicability of this idea, it has been tested on a representative power system problem, i.e. Single machine infinite bus. However, this idea can be extended to other power system problems such as multi-machine and multi area LFC problems.

\section{THEORY OF SLIDING MODE CONTROL}

The fundamental theory of SMC may be found in [1-3]. Different control goals such as stabilization, tracking, regulation can be achieved using SMC by the proper design of the sliding surface. The discussion here will be limited to the regulation problem where the objective is to keep specified states as close to zero as possible. A block diagram of the SMC for the regulation problem is shown in Figure 1. The control law is a linear state 
feedback whose coefficients are piecewise constant functions. Consider the linear time-invariant controllable system given by

$\dot{X}(t)=A X(t)+B U(t)$

Where

$X(t) n$-dimensional state vector

$U(\mathrm{t}) m$-dimensional control force vector

A $\quad n \times n$ system matrix

$B \quad n \times m$ input matrix

The SMC control laws for the system of (1) are given by

$u_{i}=-\psi_{i}^{T} X=-\sum_{j=1}^{n} \psi_{i j} x_{j} ; i=1,2, \ldots, m$

Where the feedback gains are given as

$\psi_{i j}=\left\{\begin{array}{l}\left.\alpha_{i j}, \text { if } x_{i} \sigma_{j}\right\rangle 0 ; i=1, \ldots ., m \\ -\alpha_{i j} \text { if } x_{j} \sigma_{i}\langle 0 ; j=1, \ldots ., n\end{array}\right.$

and

$$
\sigma_{i}(X)=C_{i}^{T} X=0, \quad i=1, \ldots, m
$$

Where $C_{i}$ 's are the switching vectors which are selected by pole placement or linear optimal control theory. 


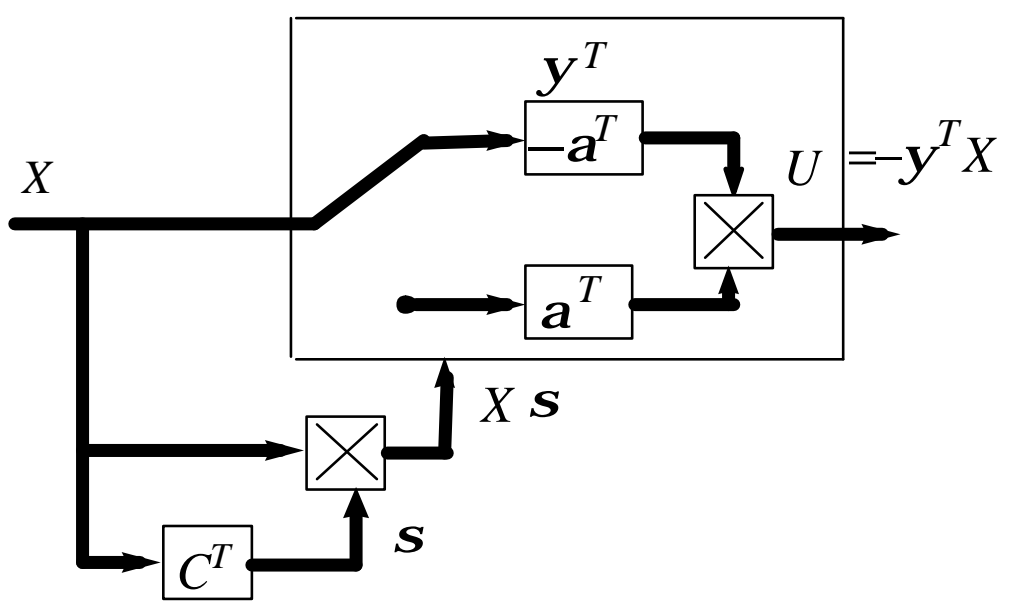

Figure 1: Block diagram of sliding mode controller (SMC)

The design procedure for selecting the constant switching vectors $C_{i}$ using pole placement is described below.

Stepl: Define the coordinate transformation

$Y=M X$

such that

$$
M B=\left[\begin{array}{l}
0 \\
\cdots \\
B_{2}
\end{array}\right]
$$

where $M$ is a nonsingular $n \times n$ matrix and $B_{2}$ is a nonsingular $m \times m$ matrix.

From (4) and (5)

$$
\dot{Y}=M \dot{X}=M A M^{-1} Y+M B U
$$

Equation (6) can be written in the form 


$$
\left[\begin{array}{l}
\dot{Y}_{1} \\
\dot{Y}_{2}
\end{array}\right]=\left[\begin{array}{l}
A_{11} A_{12} \\
A_{21} A_{22}
\end{array}\right]\left[\begin{array}{l}
Y_{1} \\
Y_{2}
\end{array}\right]+\left[\begin{array}{l}
0 \\
B_{2}
\end{array}\right] U
$$

where $A_{11}, A_{12}, \quad A_{21}, \quad A_{22}$ are respectively $(n-m) \times(n-m),(n-m) \times m, m \times(n-m)$ and $(m \times m)$ submatrices. The first equation of (7) together with (3) specifies the motion of the system in the sliding mode that is

$$
\begin{aligned}
& \dot{Y_{1}}=A_{11} Y_{1}+A_{12} Y_{2} \\
& \sum(Y)=C_{11} Y_{1}+C_{12} Y_{2}
\end{aligned}
$$

where $C_{11}$ and $C_{12}$ are $m \times(n-m)$ and $(m \times m)$ matrices, respectively satisfying the relation

$$
\left[C_{11} C_{12}\right]=C^{T} M^{-1}
$$

Equations (8) and (9) uniquely determine the dynamics in the sliding mode over the intersection of the switching hyperplanes

$\sigma_{i}(X)=C_{i}^{T} X=0, \quad i=1, \ldots ., m$

The subsystem described by (8) may be regarded as an open loop control system with state vector $Y_{1}$ and control vector $Y_{2}$ being determined by (9), that is

$$
Y_{2}=-C_{12}^{-1} C_{11} Y_{1}
$$

Consequently, the problem of designing a system with desirable properties in the sliding mode can be regarded as a linear feedback design problem. Therefore, it can be assumed, without loss of generality, that $C_{12}=$ identity matrix of proper dimension.

Step 2: Equations (8) and (11) can be combined to obtain

$$
\dot{Y}_{1}=\left[A_{11}-A_{12} C_{11}\right] Y_{1}
$$

Utkin and Yang [16] have shown that if the pair (A, B) is controllable, then the pair $\left(A_{11}, A_{12}\right)$ is also controllable. If the pair $\left(A_{11}, A_{12}\right)$ is controllable, then the eigenvalues of the 
matrix $\left[A_{11}-A_{12} C_{11}\right]$ in the sliding mode can be placed arbitrarily by suitable choice of $C_{11}$. The feedback gains $\alpha_{i j}$ are usually determined by simulating the control system and trying different values until satisfactory performance is obtained.

\section{ADAPTIVE SLIDING MODE CONTROLLER}

The proposed SMC involves the following steps:

1) Generating data for the SMC gains that corresponds to different operating points using genetic algorithms.

2) Training and testing of the neural network to perform the nonlinear mapping between the operating points and SMC feedback gains.

3) Online implementation of the proposed SMC.

These steps are described in the following subsections:

\section{A. Data Generation Using Genetic Algorithms (GA)}

Genetic algorithms are directed random search techniques which can find the global optimal solution in complex multidimensional search spaces [17]. GA employs different genetic operators to manipulate individuals in a population of solutions over several generations to improve their fitness gradually. Normally, the parameters to be optimized are represented in a binary string. To start the optimization, GA use randomly produced initial solutions created by random number generator. This method is preferred when a priori knowledge about the problem is not available.

The flow chart of a simple GA is shown in Figure 3. There are basically three genetic operators used to generate and explore the neighborhood of a population and select a new generation. These operators are selection, crossover, and mutation. After randomly generating the initial population of say $N$ solutions, the GAs uses the three genetic operators to yield $N$ new solutions at each iteration. In the selection operation, each solution of the current 
population is evaluated by its fitness normally represented by the value of some objective function, and individuals with higher fitness value are selected. Different selection methods such as stochastic selection or ranking-based selection can be used.

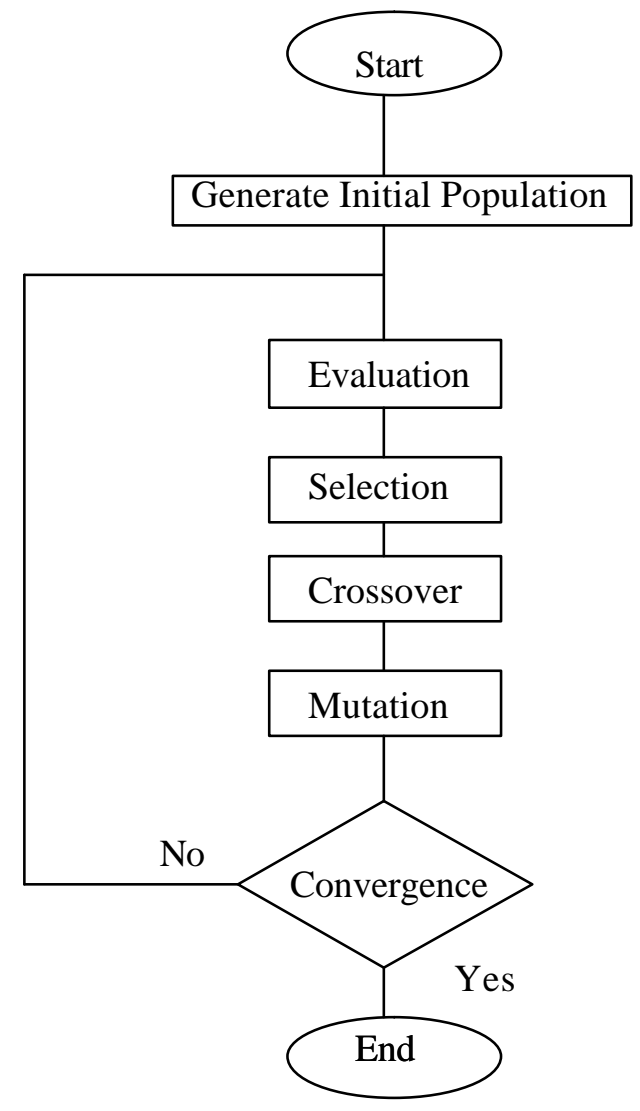

Figure 3: Flow-chart of GA

The crossover operator works on pairs of selected solutions with certain crossover rate. The crossover rate is defined as the probability of applying crossover to a pair of selected solutions. There are many ways of defining this operator. The most common way is called the one-point crossover which can be described as follows. Given two binary coded solutions of certain bit length, a point is determined randomly in the two strings and corresponding bits are swapped to generate two new solutions. Mutation is a random alteration with small probability of the binary value of a string position. This operation will prevent GA from being trapped in a local minimum. The fitness 
evaluation unit in the flow chart acts as an interface between the GA and the optimization problem. Information generated by this unit about the quality of different solutions is used by the selection operation in the GA. The algorithm is repeated until a predefined number of generations have been produced.

The use of GA to generate SMC feedback gains for different operating points is described by the following steps [12]:

1) Generate randomly a set of possible feedback gains.

2) Evaluate a performance index when the system is subjected to a change in the operating point for all possible feedback gains generated in step 1.

3) Use genetic operators (selection, crossover, mutation) to produce new generation of feedback gains.

4) Evaluate the performance index in step 2 for the new generation of feedback gains. Stop if there is no more improvement in the value of the performance index or if certain predetermined number of generation has been used, otherwise go to step 3 .

\section{B. Training and Testing of the Neural Network (NN)}

A multilayer neural network is a layered network consisting of an input layer, an output layer, and one or more hidden layers. Each layer consists of a set of neurons which are fully connected to the neurons in the next layer. The connections have multiplying weights associated with them. The number of neurons and hidden layers is problemdependent. However, it has been proved that one hidden layer can perform any nonlinear mapping and no more than two hidden layers are needed [15]. A multilayer feedforward neural network with one hidden layer is shown in Figure 4. 


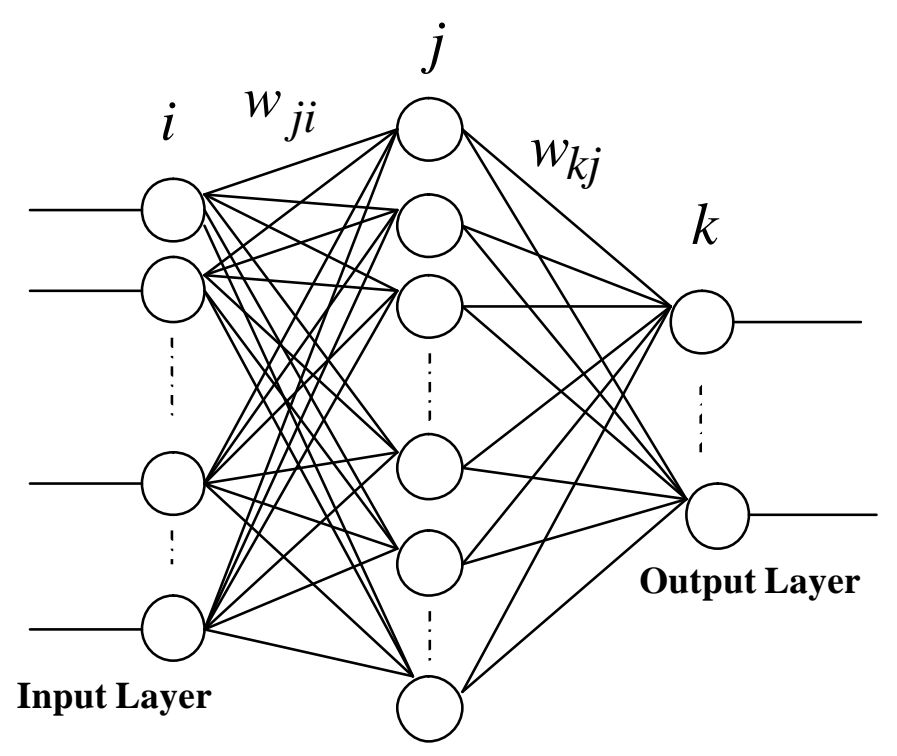

Hidden Layer

Figure 4: A multilayer feedforward neural network

The connection weights between the neurons and thresholds are determined using the generalized delta rule [18].

The process of determining the weights is called training or learning process. The training process requires a set of input and output patterns. The patterns are fed into the neural networks. The neurons in the input layer receive input signals, the activation signals propagate forward, through the hidden layer(s), to the output layer. The output layer then gives the desired output. The network learns by comparing its output of each input pattern with the actual output of that pattern. The error (the difference between the actual outputs and the predicted outputs of the network) is calculated and propagated backwards from the output to the hidden layer to the input. This is done by minimizing the error function:

$E=\sum_{p} E_{p}=\frac{1}{2} \sum_{p} \sum_{k}\left(t_{k}^{(p)}-y_{k}^{(p)}\right)^{2}$

where $t_{k}$ is the actual output and $y_{k}$ is the predicted output of the neural network. 
The inputs to the neural network used for the adaptive SMC corresponds to the system operating points while the outputs generated by the NN represent the SMC feedback gains. The training of the NN is performed using the data generated by the GA. Normally, the data is divided into two parts; one for training and the other for testing.

\section{Online Implementation of the Proposed SMC}

The block diagram of the proposed adaptive SMC is shown in Figure 6. As the operating point changes, the neural network will adaptively produce new feedback gains suitable for this new operating point.

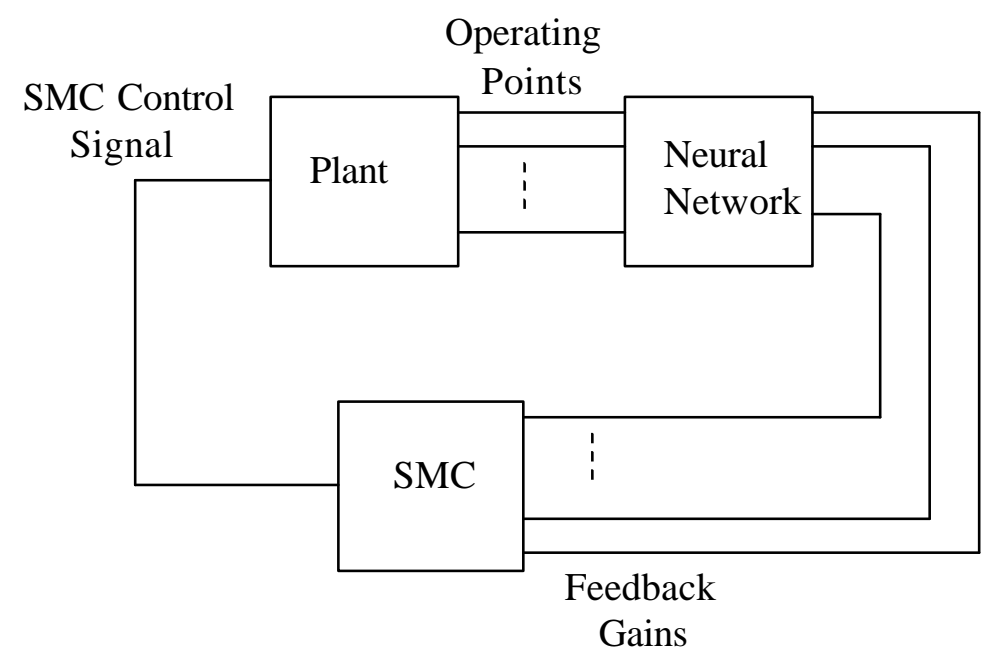

Figure 5: Block diagram of the proposed SMC

\section{SIMULATION RESULTS}

The proposed adaptive SMC is applied to the design of a power system stabilizer (PSS) of a single machine power system model where the system model is a function of the operating point defined by active and reactive powers (P, Q). The need for adaptive SMC comes from the fact that the model discussed operates over a wide range of operating points, some of which are unstable. Thus, no single SMC with fixed feedback gains is sufficient for the 
entire operation. Figure 6 shows the block diagram of the linearized power system model for low-frequency oscillation studies. The dynamic model in state-variable form can be obtained from the transfer function model and is given as [19]

$$
\dot{X}(t)=A X(t)+B u(t)+F d(t)
$$

where

$$
X(t)=\left[\begin{array}{llll}
\Delta \omega(t) & \Delta \delta(t) & \Delta e_{q}^{\prime}(t) & \Delta e_{f d}(t)
\end{array}\right]^{T},
$$$$
u(t)=u_{(\operatorname{from} S M C)}, d(t)=\Delta T_{m}(t)
$$

$A=\left[\begin{array}{cccc}-\frac{D}{M} & -\frac{K_{1}}{M} & -\frac{K_{2}}{M} & 0 \\ \omega_{0} & 0 & 0 & 0 \\ 0 & -\frac{K_{4}}{T_{d 0}^{\prime}} & -\frac{1}{T_{d 0}^{\prime} K_{3}} & \frac{1}{T_{d 0}^{\prime}} \\ 0 & -\frac{K_{4} K_{5}}{T_{A}} & -\frac{K_{4} K_{6}}{T_{A}} & -\frac{1}{T_{A}}\end{array}\right]$

$B=\left[\begin{array}{llll}0 & 0 & 0 & \frac{K_{A}}{T_{A}}\end{array}\right]^{T}$

$F=\left[\begin{array}{llll}\frac{1}{M} & 0 & 0 & 0\end{array}\right]^{T}$

$\omega$ is the rotor speed $(\mathrm{rad} / \mathrm{sec}), \delta$ is the machine shaft angular displacement (degree), $D$ is the damping coefficient, $M$ is the inertia constant, $e_{q}^{\prime}$ is the voltage proportional to the field flux linkages of machine, $e_{f d}$ is the generator field voltage, $K_{1}-K_{6}$ are constants of the linearized model, $K_{A}$ is the automatic voltage regulator gain, $T_{A}$ is the automatic voltage regulator time constant (sec), $T_{d o}^{\prime} \mathrm{d}$-axis transient open circuit time constant. The control objective in the PSS problem is to keep the change in frequency $(\Delta \omega)$ as close to zero as possible when the operating point changes by manipulating the input $(u)$. For this plant, the pair $(A, B)$ has been found to be controllable. 


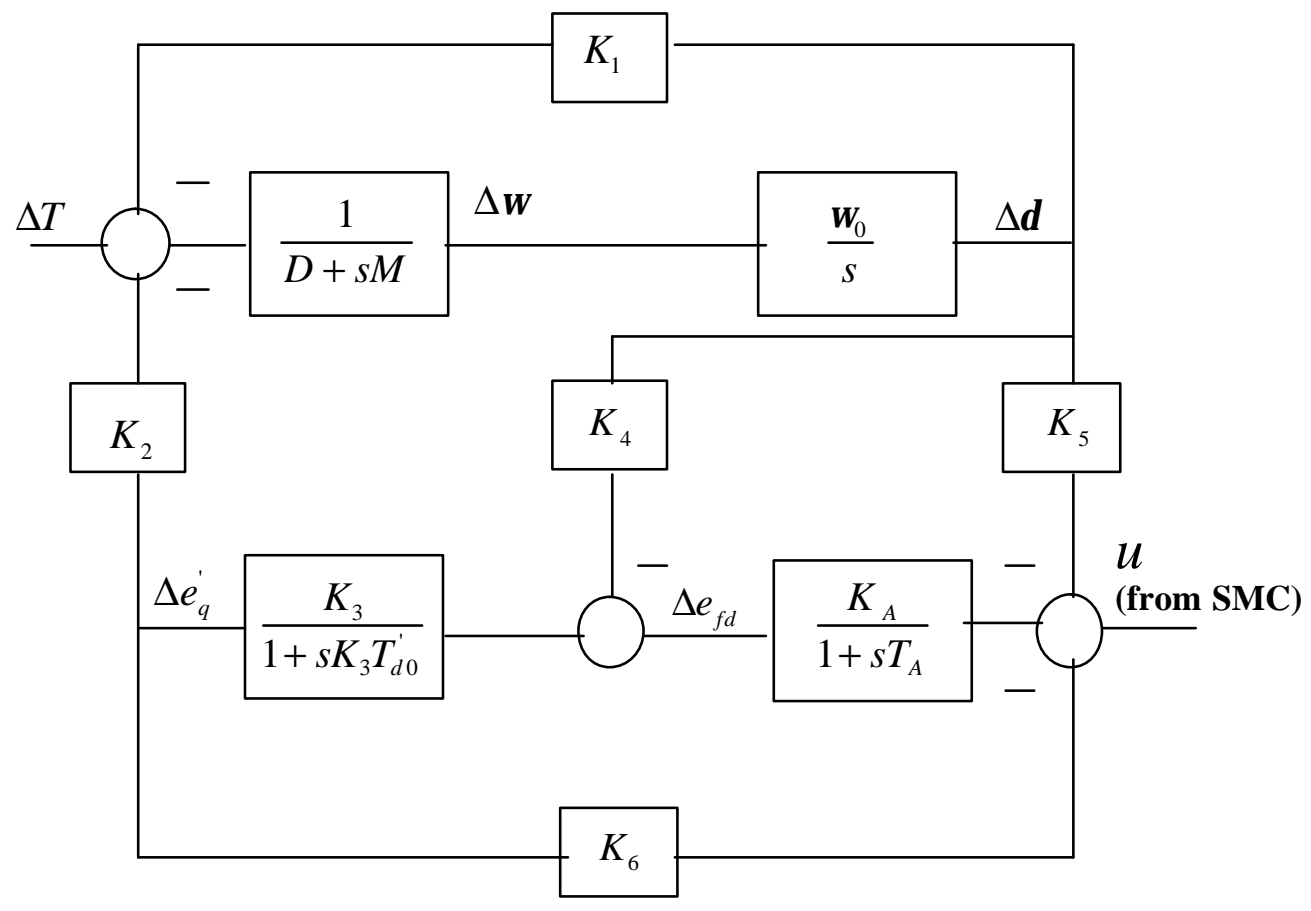

Figure 6: Block diagram of a single machine power system model

Following the GA design procedure described in Section III.A, crossover and mutation probabilities as well as population size of $0.7,0.001$, and 35 are used respectively to get the optimal SMC gains $\left(\alpha_{1}\right.$ and $\left.\alpha_{2}\right)$ corresponding to different operating points in the range ( $P$ from 0.1 to $1 \mathrm{p} . \mathrm{u}$ and $Q$ from-1 to $1 \mathrm{p}$.u) The performance index given by:

$$
J=\int_{0}^{\infty} \Delta \omega^{2}(t) d t
$$

will be minimized using GA. The minimization of this performance index will keep the change in frequency $(\Delta \omega)$ as close to zero as possible regardless of the control effort $(u)$. The behavior of the performance index is shown in Figure 7 where it can be seen that the convergence is very fast. 


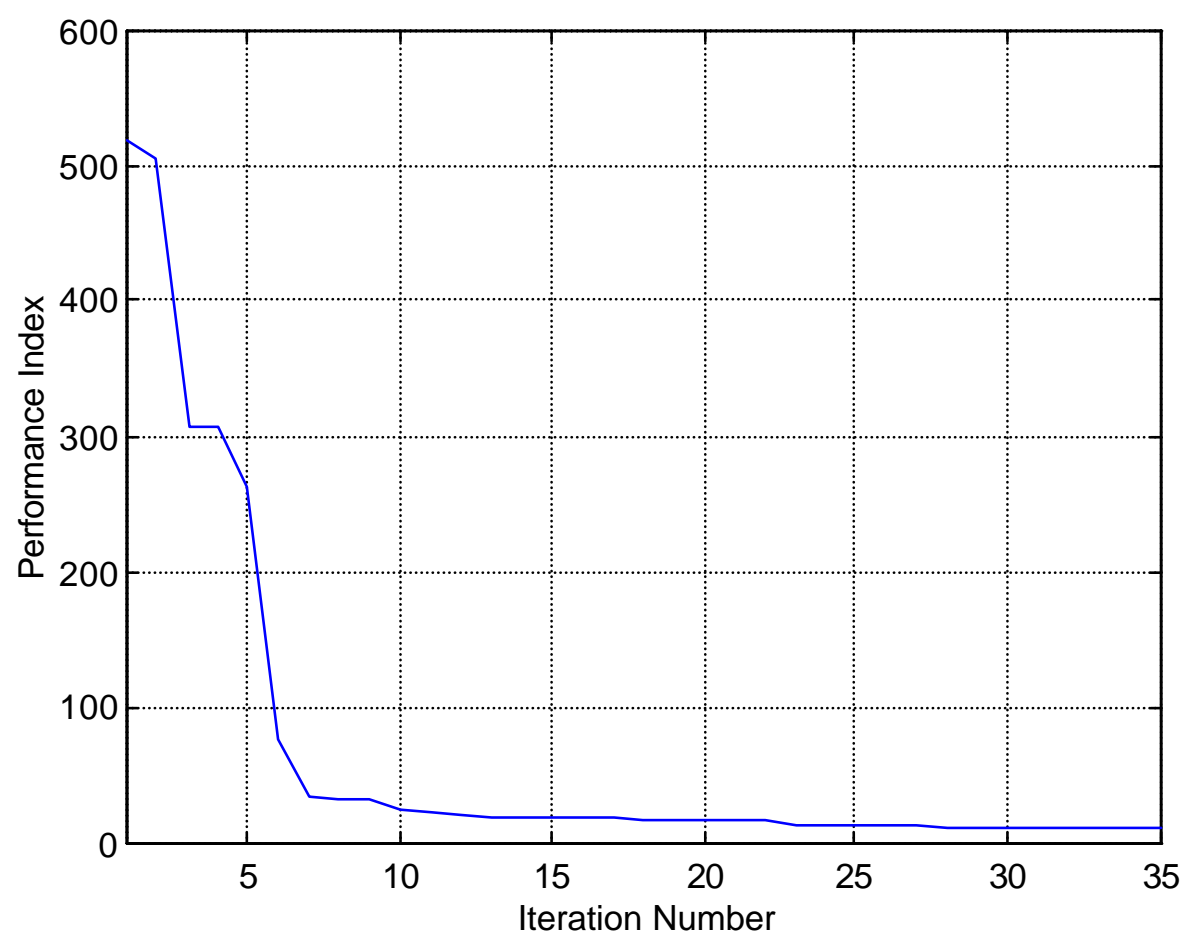

Figure 7: Values of the Performance Index (J)

In the NN training process 210 operating points generated by changing $P$ from 0.1 to 1.0 (per unit) and $Q$ from 1 to 1 (per unit) which represent the practical operating range of the studied system, are used. The change has been made in steps of 0.1 . In practice, any step change can take place. The neural network used has two inputs $(P, Q)$, two outputs $\left(\alpha_{1}\right.$ and $\left.\alpha_{2}\right)$, and 30 neurons in the hidden layer. Figure 8 shows the online implementation of the proposed adaptive SMC. When the operating point $(P, Q)$ changes, the trained neural network will adaptively produce new feedback gains $\left(\alpha_{1}\right.$ and $\left.\alpha_{2}\right)$ suitable for this new operating point. 


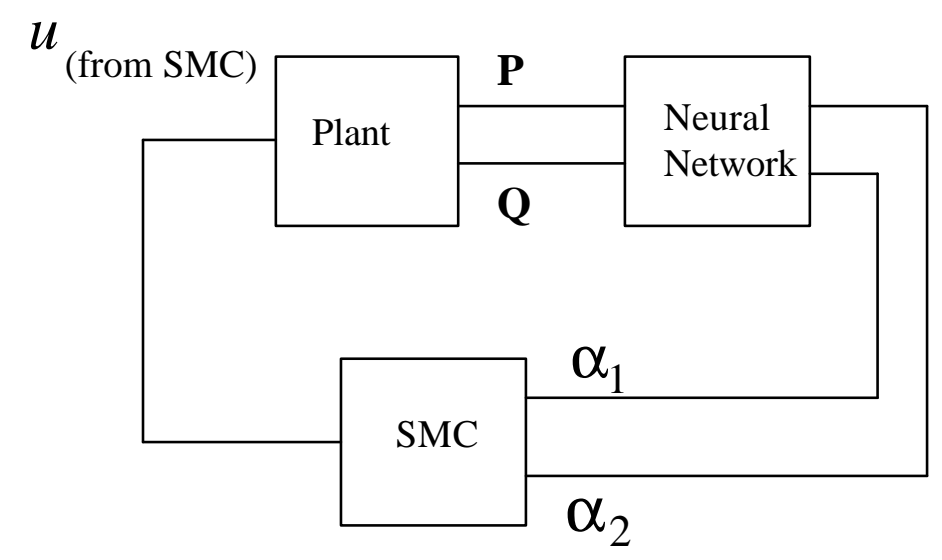

Figure 8: On-line implementation of the adaptive SMC.

The results of training and testing the neural network are shown in Figure 9 and Figure 10. The first 80 percent of data were used for training and the rest 20 percent were used for testing of the neural network. Findings indicate good agreement between the actual feedback gains (generated by GA) and outputs of the neural network.

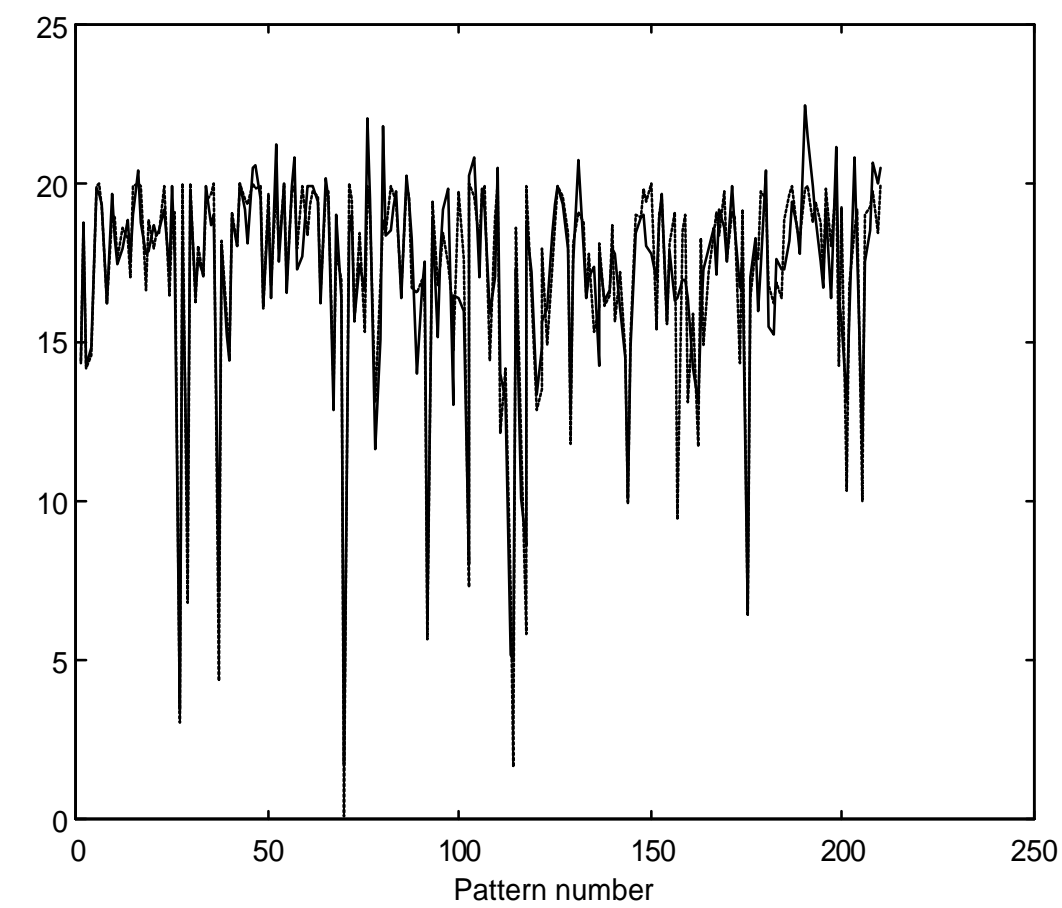

Figure 9: Actual (-) and predicted (....) values of $\alpha_{1}$ 


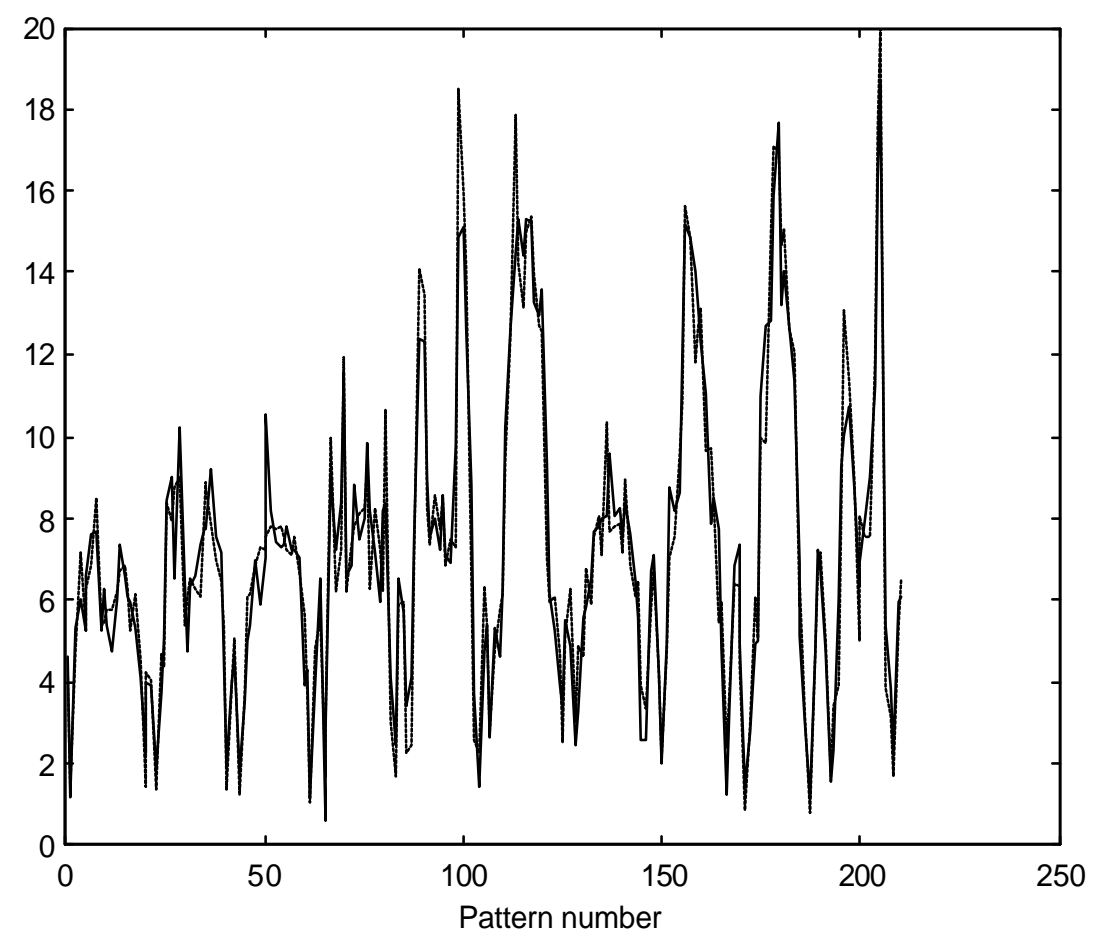

Figure 10: Actual (-) and predicted (...) values of $\alpha_{2}$

For the operating point of $(P=0.1, Q=1.0)$, a fixed variable structure controller for the above system has been designed. To reduce the complexity of the SMC, the two states $\Delta \omega$ and $\Delta \delta$ are used for feedback. The switching vector is given to be [20]

$$
C=\left[\begin{array}{llll}
-30000 & -97.2134 & 107.0026 & 1
\end{array}\right]^{T}
$$

and the feedback gains obtained using genetic algorithms are

$\alpha_{1}=18.5109 \quad, \alpha_{2}=4.2116$

Figure 11 shows the simulation results of the change in frequency $(\Delta \omega)$ when the operating point of the systems changes from $(P=0.1, Q=1.0)$ to $(P=0.3, Q=-0.9)$ at time 10 seconds which has not been used in the training set. This figure demonstrates the effectiveness of the adaptive SMC in damping the frequency oscillations. On the other hand, Figure 12 shows the change in the torque angle when using the fixed and adaptive SMC. 


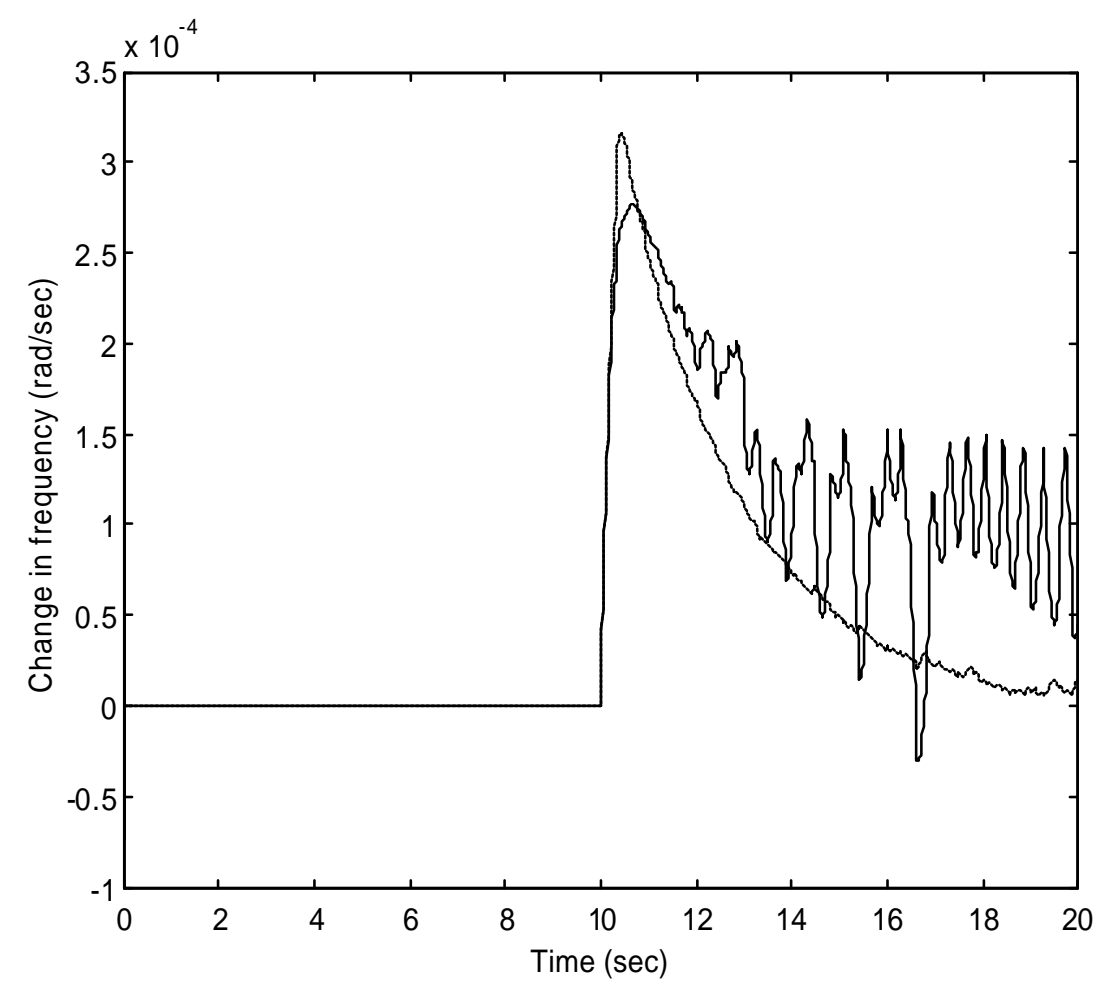

Figure: 11 Change in frequency ( $\Delta \omega$ ) for fixed and adaptive SMC gains: (-) for fixed, (...) for adaptive

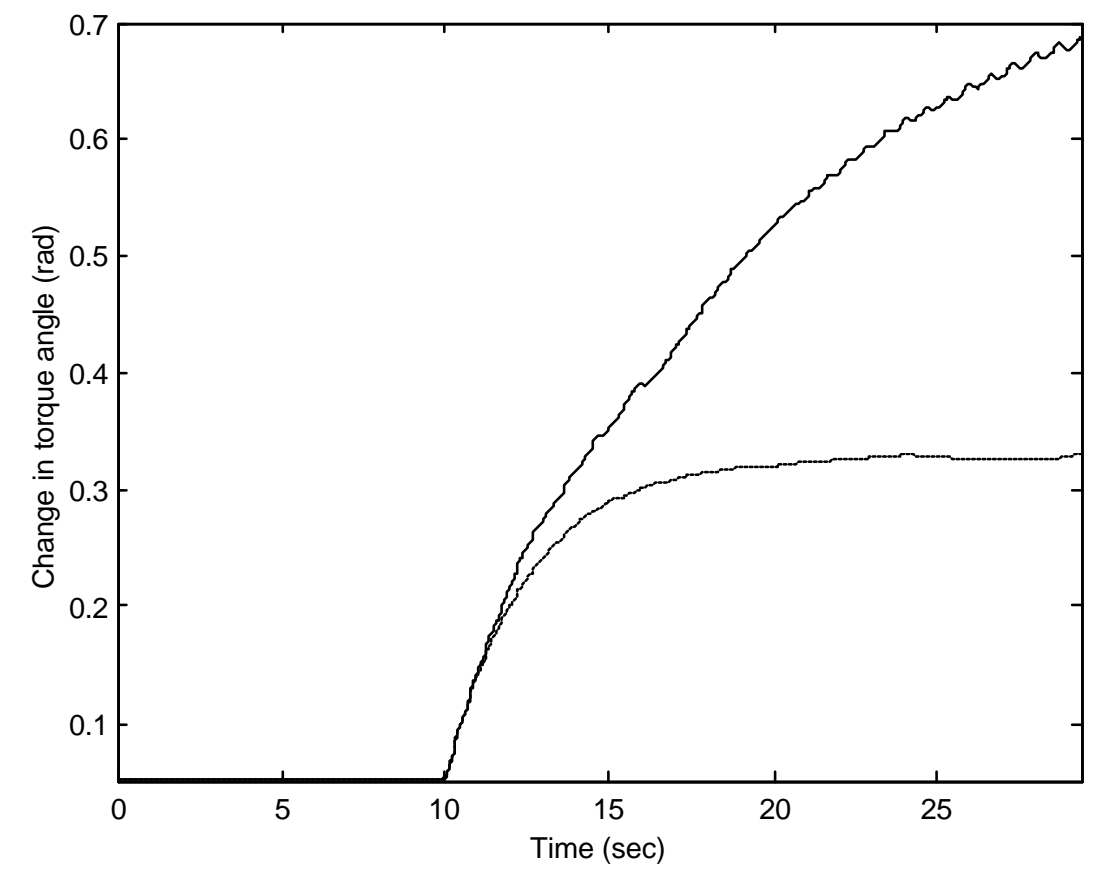

Figure 12: Change in torque angle $(\Delta \delta)$ for fixed and adaptive SMC gains: (-) for fixed, (...) for adaptive 
It is quite clear that the adaptive SMC drives the torque angle to its steady state value much faster than the fixed SMC. Figure 13 shows the control efforts of the fixed and adaptive SMC gains. The figure clearly demonstrates the lower control effort needed for the case of adaptive SMC gains.

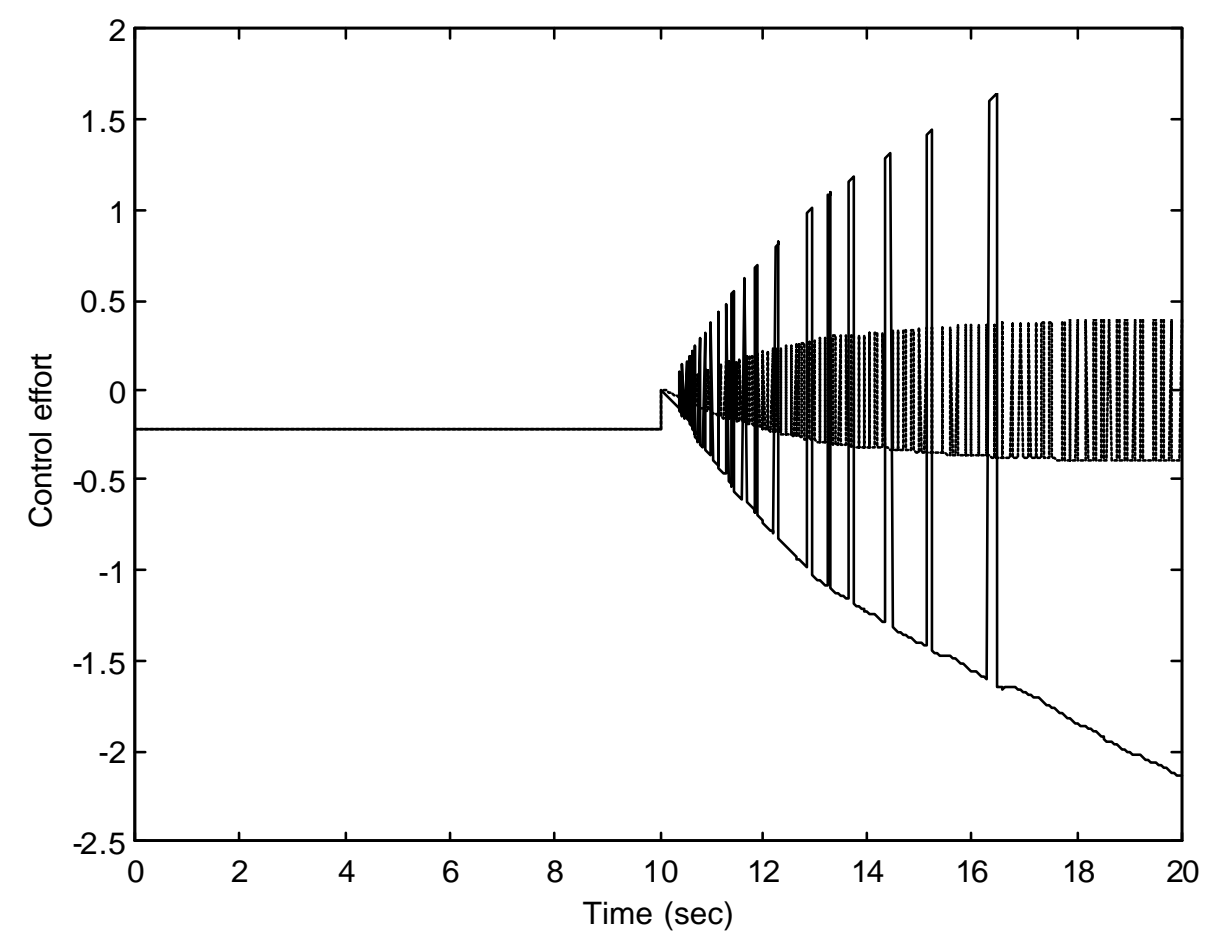

Figure 13: Control efforts for fixed and adaptive SMC gains: (-) for fixed, (...) for adaptive

\section{CONCLUSIONS}

In this paper, an adaptive neural network based SMC has been developed for a PSS of a single machine power system. The use of adaptive output feedback is motivated by the fact that the single machine power system operates over a wide range of operating conditions and hence no single SMC gains are sufficient for the entire operation. The neural network is used to adaptively predict the suitable SMC gains for any operating point. The training data for the neural network has been generated using genetic algorithms. Simulation results indicate 
that fixed SMC can not perform satisfactorily for a wide range of operating points, while the controller performance is greatly improved by the use of adaptive SMC.

\section{ACKNOWLEDGMENT}

The authors acknowledge King Fahd University of Petroleum \& Minerals (KFUPM) for the support they received.

\section{REFERENCES}

[1] K. Young, V., Utkin, and U., Ozguner, “A Control engineer's guide to sliding mode control”, IEEE Transactions on Control Systems Technology, vol 7, no. 3, 1999, pp. 328-342.

[2] J. Y. Hung, W., Gao, and J. C., Hung, "Variable structure control: A survey. IEEE Transaction on Industrial Electronics, vol. 40, no. 1, 1993, pp. 2-22.

[3] R. DeCarlo, S. Zak, and G. Matthews, "Variable structure control of nonlinear multivariable systems: A Tutorial', Proceedings of the IEEE, vol 76, no.3, 1988, pp. 212-232.

[4] P. Liutanakul, S. Pierfederici, and F. Tabar, "Nonlinear control techniques of a controllable rectifier/invertermotor drive system with a small DC-link capacitor", Energy Conversion and Management, vol 49, 2008, pp. 3541-3549.

[5] I. Baik, K. Kim, and M. Youn, “ Robust nonlinear speed control of PM synchronous motor using boundary layer integral sliding mode control technique”, IEEE Transactions on Control Systems Technology, vol. 8, no. 1, 2000, pp. 47-54.

[6] I. Munteanu, S. Bacha, A. Bratcu, J. Guiraud, and D. Roye, "Energy-reliability optimization of wind energy conversion systems by sliding mode control”, IEEE Transactions on Energy Conversion, vol 23, no. 3, 2008, pp. 275-985.

[7] A.Y. Sivarmakrishnan, M.A Hariharan and M.C. Srisailam, "Design of variable structure load frequency controller using pole assignment technique”, Int. J. Control, Vol. 40, No. 3, 1984, pp. 487-498. 
[8] A.S. Murty, S. Parameswaran and K. Ramar, "Design of variable structure stabilizer using pole assignment technique", Electric Machines and Power Systems, Vol.26, No. 3, 1998, pp. 185-206.

[9] P. Balasubramanyam, A. Murthy and S. Parameswaran, "Design of variable structure controller for static VAR compensator", Electric Machines and Power Systems, Vol. 26, No. 4, 1998, pp. 431-450.

[10] R. J. Fleming and Jun Sun, “An optimal variable structure stabilizer for a synchronous generator,” International Journal of Power and Energy system s, vol. 12, No. 1, 1992.

[11] C. Bhattacharya, M. Kothari, and J. Nanda, "Design of discrete mode variable structure power system stabilizers," International Journal Electric Power and Energy Systems, vol 17, No. 6, pp. 399-406, 1995.

[12] Z. Al-Hamouz and H. Al-Duwaish, "A new load frequency variable structure controller using genetic algorithms ", Electric Power Systems Research, Vol. 55, 2000, pp. 1-6.

[13] Z. Al-Hamouz, N. AlMusabi, H. Al-Duwaish and S. Al-Baiyat, "On the Design of Variable Structure Load Frequenc y Controllers by Tabu Search Algorithm: Application to Nonlinear Interconnected Models”, Electric Power Components and Systems, Vol33, 2005, pp. 1253-1267.

[14] N. Al-Musabi, Z. Al-Hamouz, H. Al-Duwaish, " Design of Variable Structure Stabilizer for a Nonlinesr Mode of SMIB System: Particle Swarm Approach", WSEAS Transactions on Power Systems, 2006, pp. 311-316.

[15] R. Lippman, "An introduction to computing with neural nets", IEEE ASSP Mag., 1987, pp. 4-22.

[16] V. Utkin and K. Yang, "Methods for constructing discontinuity planes in multidimentional variable structure systems", Autom. and Remote Control', Vol 39, 1978, pp. 1466-1470.

[17] D. Goldberg, Genetic Algorithms in Search Optimization and Machine Learning, Addison-Wesley, 1989 
[18] D. Rumelhart, G Williams and R Williams, "Learning internal representation by error propagation", Parallel-Distributed Processing, Vol. 1, MIT Press, Cambridge, MA, 1986, pp. 318-362.

[19] Y. N. Yu, Electric power system dynamics, Academic Press, New York, 1983.

[20] S. Lee and J. Park, "Design of power system stabilizer using observer/sliding mode, observer/sliding modemodel following and $H_{\infty} /$ sliding mode controllers for small-signal stability study", Electric Power and Energy Systems, Vol. 20, 1998, pp. 543-553. 